\title{
Optimal control methods for nonlinear parameter estimation in biophysical neuron models
}

\author{
Nirag Kadakia ${ }^{1,2,3^{*}}$ \\ 1 Department of Molecular, Cellular, and Developmental Biology, Yale University, New \\ Haven, CT, USA \\ 2 Quantitative Biology Institute, Yale University, New Haven, CT, USA \\ 3 Swartz Foundation for Theoretical Neuroscience, Yale University, New Haven, CT, \\ USA \\ * nirag.kadakia@yale.edu
}

\begin{abstract}
Functional forms of biophysically-realistic neuron models are constrained by neurobiological and anatomical considerations, such as cell morphologies and the presence of known ion channels. Despite these constraints, neurons models still contain unknown static parameters which must be inferred from experiment. This inference task is most readily cast into the framework of state-space models, which systematically takes into account partial observability and measurement noise. Inferring only dynamical state variables such as membrane voltages is a well-studied problem, and has been approached with a wide range of techniques beginning with the well-known Kalman filter. Inferring both states and fixed parameters, on the other hand, is less straightforward. Here, we develop a method for joint parameter and state inference that combines traditional state space modeling with chaotic synchronization and optimal control. Our methods are tailored particularly to situations with considerable measurement noise, sparse observability, very nonlinear or chaotic dynamics, and highly uninformed priors. We illustrate our approach both in a canonical chaotic model and in a phenomenological neuron model, showing that many unknown parameters can be uncovered reliably and accurately from short and noisy observed time traces. Our method holds promise for estimation in larger-scale systems, given ongoing improvements in calcium reporters and genetically-encoded voltage indicators.
\end{abstract}

\section{Introduction}

Computations in biological neural networks are shaped both by connection topologies and the response dynamics of individual neurons $1-3$. For single neurons, a versatile and biologically realistic class of computational models is built on the Hodgkin-Huxley framework - so-called conductance-based neuron models [4]. These nonlinear dynamical systems relate the temporal evolution of the neuron membrane voltage to the flow of ionic currents into and out of the neuron. Since they model the continuous-valued membrane voltage, rather than a binary-valued train of spike events, they can produce a wide class of neuron behaviors, including subthreshold oscillations and spikes and bursts of various waveforms, among others [1. In addition, their complexity can often be tuned for the problem at hand, by appropriate simplifications of the underlying dynamical system [1]. 
Fitting such biophysically realistic neural models to data is often cast in the framework of statistical inference [5 9], which systematically takes into account i) noise in the model dynamics and in the observations, and ii) unobservability in model states. Moreover, inference procedures produce not only an optimal estimate of model unknowns, but also the distributions of these estimates around their optima, providing a measure of estimate uncertainty. Various manifestations of statistical inference have been applied in neurobiological, behavioral, and neuromorphic settings [6,7, 7,21$]$.

In many settings, the emphasis of statistical inference has been on tracking dynamical variables, such as the membrane voltage, over time. Algorithms like the Kalman filter [5, 22, or its many variants [5,5, 23,24 solve this state estimation problem recursively, by updating the optimal estimate sequentially with each subsequent observation. This is computationally cheap and memory-efficient, requiring only the estimate at the most recent timestep. But extracting time-varying quantities is only one aspect of the inference procedure, and is not usually the direct quantity of interest. Rather, it is knowledge of the fixed parameters, such as time constants, channel conductances, baseline ionic concentrations, and synaptic strengths, that is required to generate predictions to novel stimuli, informing our understanding of brain function. Recursive filtering methods break down when the neuron model parameters are unknown, since the model dynamics, which together with the data determine subsequent estimates, are not fully specified. One approach is to "promote" parameters to dynamical states in their own right, but with trivial dynamics - this keeps the parameters approximately constant, while allowing enough stochasticity to improve estimates [5]25]. A more sophisticated method combines particle filtering and parameter inference into an expectation-maximization algorithm that sequentially updates state and parameter estimates in alternating fashion 5.26 .

An alternative to all of these sequential filtering approaches is the direct optimization of a posterior distribution over, jointly, the states at all timepoints and the unknown parameters. Optimization-based methods have been used to effectively estimate linear parameters in neural models 6, 26, 30, as well as to uncover nonlinear parameters in nonlinear and even chaotic models [7, 17, 20,31,37. In this article, we build on some of these approaches to present a new method for joint state and parameter estimation in biophysical neural models. Our emphasis is on strongly nonlinear (including chaotic) models, sparse observations, unknown nonlinear parameters, and extreme nonconvexity in the state and parameter manifold. We combine two ideas which have recently been investigated in nonlinear systems estimation: chaotic synchronization [38 40], and homotopy continuation 7, 33, 41, 42]. Our approach is predicated on a previous observation that, by coupling measured data directly into the system dynamics, cost manifolds over the unknown states and parameters become highly regularized 37,40 . We extend this notion into an optimal control framework, treating the coupling strength as a control parameter which we optimize using the Pontryagin minimum principle 43 . What results is a new dynamical system that represents the evolution of the optimal state estimate in time. We present an algorithm to simultaneously find the integral curves of these "estimation dynamics" and determine the unknown parameters from noisy data. We illustrate our algorithm using synthetic data from both a sparsely observed canonical choatic attractor and a biophysical HH-type model, comparing to the prior techniques [33 40].

The results are organized as follows. We give an overview and example of the "nudging" technique of chaotic synchronization, upon which our ideas are predicated. We then discuss a previously-proposed method to incorporate parameter estimation into nudging, and then present our main algorithm. We illustrate our technique with numerical experiments in the canonical chaotic Lorenz96 system 44 and a biophysical neuron model, respectively. 


\section{Results}

\section{Nudging synchronization for estimating dynamical states}

We first consider the problem of estimating the hidden dynamical states of a system with known dynamics from noisy observations, focusing on a simple technique which will form the basis for our proposed approach. The system evolves either deterministically via the ODE dynamics

$$
\dot{\mathbf{x}}(t)=\mathbf{f}(\mathbf{x}(t), \boldsymbol{\Theta})
$$

or stochastically under the Langevin dynamics

$$
\dot{\mathbf{x}}(t)=\mathbf{f}(\mathbf{x}(t), \boldsymbol{\Theta})+\eta(t),
$$

where $\eta(t)$ is uncorrelated Gaussian noise, and for now we assume that all model parameters $\boldsymbol{\Theta}$ are known. The system is sparsely observed, so the dimension $L$ of the observation vector $\mathbf{y}(t)$ is generally less than that of the $D$-dimensional state space $\mathbf{x}(t)=\left[x_{1}(t), x_{2}(t), \ldots, x_{D}(t)\right]$, e.g. $L<D$. For notational simplicity, throughout we represent $\mathbf{y}(t)$ as an $D$-dimensional vector with nonzero elements only in its $L$ observed elements. An unsophisticated but straightforward and computationally attractive way to infer the evolution of both the observed and unobserved variables is by dynamically coupling the system to the measurements, effectively controlling the system dynamics with data $38,40,45,47$. To do this, we redefine the dynamics as:

$$
\dot{\mathbf{x}}(t)=\mathbf{f}(\mathbf{x}(t), \boldsymbol{\Theta})+\mathbf{U} \cdot(\mathbf{y}(t)-\mathbf{H x}(t)) \equiv \mathbf{f}_{\text {cont }}(\mathbf{x}(t), \mathbf{y}(t), \boldsymbol{\Theta})
$$

where $\mathbf{U}$ is a constant, diagonal matrix with nonzero elements $u_{l l}$ for observed states $l$, and $\mathbf{H}$ projects the state space $x$ onto the observed subspace $\mathbf{y}$ - i.e. both $\mathbf{U}$ and $\mathbf{H}$ are $D \mathrm{x} D$ but have rank $L$. The initial states $\mathbf{x}\left(t_{0}\right)$, can be initialized randomly, and the dynamics Eq 3 are simply integrated forward [45. Information passes from the data to the observed $L$ states through the linear control term $\mathbf{U}(\mathbf{y}-\mathbf{H x})$, then from observed to the $D-L$ unobserved states through the coupled dynamical equations (Fig 1 A). Information therefore passes from data to all model states, observed or not. The strength of the nonzero control terms $u_{l l}$ should be set to a value large enough to synchronize the system to the data, but not so large that the noise in the observations is magnified 46. In the geophysics literature, this technique is known as "nudging" 45.46. Those familiar with linear filtering or control theory will recognize that the control term, a linear scaling of the error between data and estimate, is analogous to the innovation term in the Kalman filter [5,22. However, the Kalman gain evolves in time and is chosen to minimize the residual estimation error at each time step, while $\mathbf{U}$ is constant and prescribed upfront. Though the Kalman prescription is more principled than the choice of $\mathbf{U}$, there is a tradeoff in the computational costs of storing and manipulating large convariance matrices, not required here. As will be discussed below, the more pressing issue is that sequential estimators such as both nudging and the Kalman filter are not directly suited for the estimation of static parameters.

We illustrate nudging synchronization in the chaotic Lorenz96 [44] system in 5 dimensions:

$$
\begin{array}{r}
\dot{x}_{d}=\left(x_{d+1}-x_{d-2}\right) x_{d-1}-x_{d}+F \\
d=1, . ., 5 ; \quad x_{d+5}=x_{d}
\end{array}
$$

The Lorenz96 system contains a single parameter $F$, which for values $\sim 8$ render the dynamics chaotic. We assume that only $x_{1}$ and $x_{4}$ are observed, with Gaussian 
A

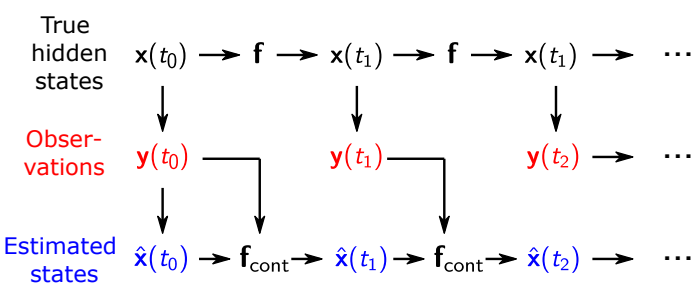

C

No synchronization

$u_{11}=u_{44}=0$

estimated states

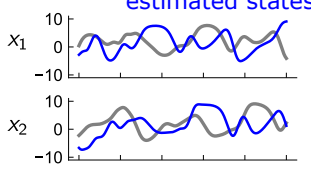

$x_{2}{ }_{-10}^{10}+100$

$x_{-10}^{10} \times 100$

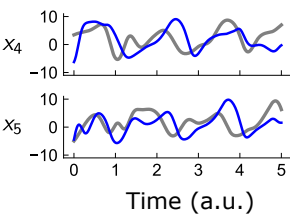

D $u_{11}=u_{44}=5.0$
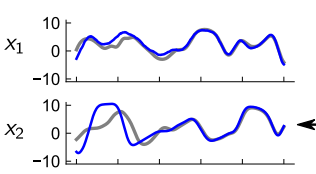

$x_{2}$

10
$\times 3$
-10
10

$x_{4}$

10
-10
-10

$x_{5}$
Nudging synchronization

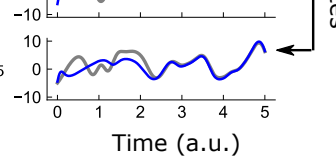

B

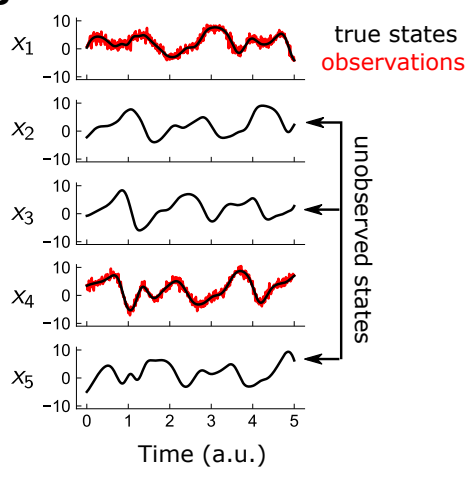

$\mathbf{E}$

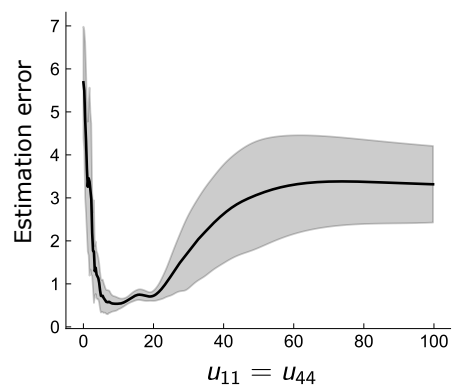

Fig 1. Nudging synchronizes estimate to observations with lax parameter tuning A: Hidden states $\mathbf{x}$ evolve temporally under the controlled dynamics via $\dot{\mathbf{x}}=\mathbf{f}_{\text {cont }}(\mathbf{x})$, where $\mathbf{f}_{\text {cont }}$ is the sum of model dynamics and an external data-driven control term. The control feeds back the difference between $\mathbf{x}$ and the observations $\mathbf{y}$, for measured states; it is absent in unmeasured states. B: Ground-truth trajectories and observations Lorenz96 system in 5 dimensions; only $x_{1}$ and $x_{4}$ are observed.

Measurements obtained by adding uncorrelated noise with $\sigma=1$ at each timestep. C and D: True (black) and estimated (blue) trajectories of the Lorenz96 system, for no nudging control $(\mathrm{C})$ and with $x_{1}$ and $x_{4}$ controlled with strength $U_{11}=U_{44} \equiv u=5$ (D). The controlled system synchronizes closely to ground truth for both the observed unobserved variables. E: Mean-squared error $\frac{1}{N D} \sqrt{\sum_{d, n}\left(x_{d}\left(t_{n}\right)-\hat{x}_{d}\left(t_{n}\right)\right)^{2}}$ of estimates as a function of control strength; near-minimal errors are achieved over a relatively wide range of $u$. 
measurement noise, so

$$
\begin{gathered}
\mathbf{y}=\mathbf{H x}+\mathbf{R}(t) \\
H_{11}=H_{44}=1 \\
R_{1}(t), R_{4}(t) \sim \mathcal{N}\left(0, \sigma^{2}\right)
\end{gathered}
$$

and the remaining $H_{i j}$ are zero and $\sigma=1$. True states $\mathbf{x}$ are generated by integrating $\mathrm{Eq} 4$ numerically with a timestep of $d t=0.01$ over $t \in[0,5]$, from which observations

are generated using Eqs. 5 (Fig 1B). Using these observations y, we obtain an estimate $\hat{\mathbf{x}}$ of the true states by integrating forward the nudged dynamics, Eq 3 , with

$U_{11}=U_{44} \equiv u$ set to a fixed value, chosen between 0 and 100 . We initialize our estimate at time $t=0$ to the measured data $\mathbf{y}(0)$ for the observed variables $x_{1}$ and $x_{4}$, and uniformly between \pm 5 for the hidden variables. This chaotic system is hypersensitive to errors in the initial conditions, so without control, $u=0$, the estimated trajectory evolves in a manner quite distinct from the true model (Fig $1 \mathrm{C}$ ). For sufficient $u$, e.g. $u=5.0$, the data synchronizes the estimates of both observed and unobserved states to the model, despite large initialization errors in $\hat{\mathbf{x}}(0)$ (Fig 1D). A distinct advantage of nudging is that fine-tuning $u$ is not necessary: within a substantial window of $u$, between $\sim 5-20$, the system closely synchronizes to the true states; however, for $u$ sufficiently large, the control term now overamplifies the observation noise, degrading the estimates (Fig $1 \mathrm{E}$ ). The simplicity of nudging synchronization is apparent: it necessitates only the choice of the gain parameter $u$, which can be chosen rather loosely - and requires only a simple forward integration of the model dynamics.

\section{Parameter inference in nudging synchronization}

In the context of neuroscience, we are less interested in the time course of dynamical state variables than model parameters such as ion channel conductances and kinetic time constants $18,19,48$. One could envision a direct search over parameter space with some appropriately chosen cost function. To illustrate why a naive optimization may not be entirely straightforward, we plot the quadratic cost between the measurements and model, $C(F)=\sum_{n}\left\|\mathbf{y}\left(t_{n}\right)-\mathbf{H x}\left(t_{n} ; F\right)\right\|^{2}$, where $\mathbf{x}\left(t_{n} ; F\right)$ is the solution of the Lorenz96 model with forcing parameter $F($ Fig $2 \mathrm{~A})$ and the initial condition $\mathbf{x}\left(t_{0} ; F\right)$ is fixed to its true value. The global minimum of this cost surface for $F$ corresponds to the true parameter $F_{\text {true }}=8$, but it resides in a narrow basin of attraction and is surrounded by multiple false minima ( Fig $2 \mathrm{~A})$. This irregularity is a consequence of the highly nonlinear nature of the dynamics [37, 40], and it would be exceedingly difficult to pinpoint the global minimum with conventional optimization routines, even in this optimistic scenario in which $\mathbf{x}\left(t_{0}\right)$ is assumed known and the search space is effectively one-dimensional.

On the other hand, the cost between the measurements and the controlled dynamics is far more regular. If we instead consider $C_{u}(F)=\sum_{n}\left\|\mathbf{y}\left(t_{n}\right)-\mathbf{H x}\left(t_{n} ; F, u\right)\right\|^{2}$, where $\mathbf{x}\left(t_{n} ; F, u\right)$ is generated from the controlled dynamics Eq 3 , we find that the cost surface smooths considerably with increasing nudging strength (Fig 2B). For sufficient gain

$(u=15)$, the cost surface is fully convex, exhibiting a broad minimum around $F=7.81$. Thus, the linear control term proportional to $u$ acts to smooth the nonconvexities of the cost surface induced by the chaotic dynamics. This observation led Abarbanel et al. to propose the following constrained optimization problem [40]:

$$
\begin{array}{r}
C\left(\mathbf{x}\left(t_{n}\right), \Theta, \mathbf{U}\left(t_{n}\right)\right)=\sum_{n}\left\|\mathbf{H x}\left(t_{n}\right)-\mathbf{y}\left(t_{n}\right)\right\|^{2}+\left\|\mathbf{U}\left(t_{n}\right)\right\|^{2}, \\
\text { s.t. } \quad \dot{\mathbf{x}}\left(t_{n}\right)-\mathbf{f}_{\text {cont }}\left(\mathbf{x}\left(t_{n}\right), \mathbf{U}\left(t_{n}\right), \Theta\right)=0, \quad \forall n
\end{array}
$$


A

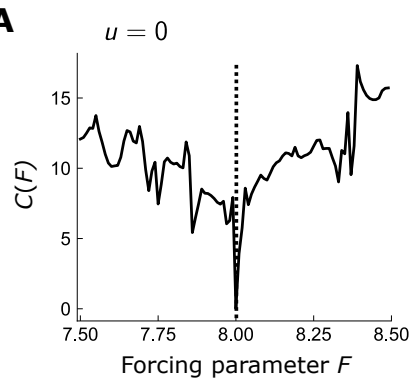

C

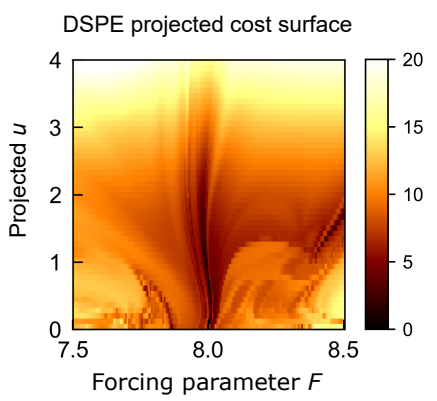

B

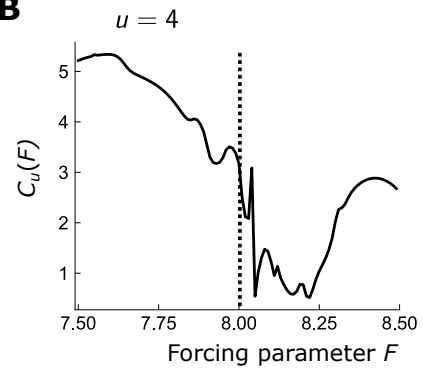

D

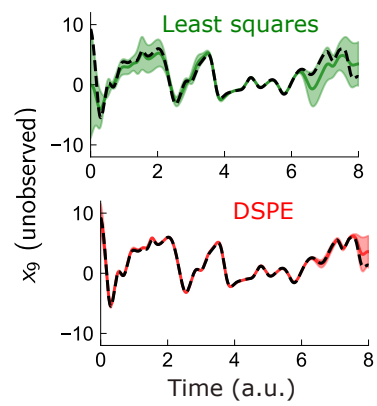

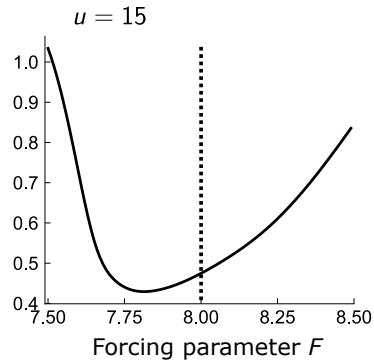

$\mathbf{E}$

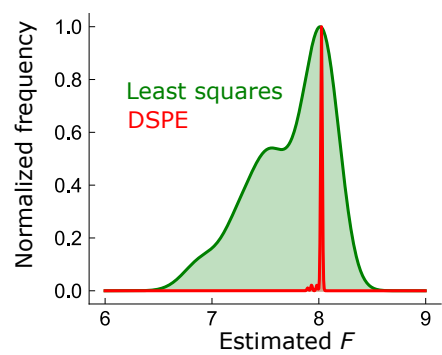

Fig 2. Constraining least-square estimates with controlled dynamics enables robust parameter estimation in chaotic models A: Squared-difference between 10D Lorenz96 model trajectory $\mathbf{x}$ and measured data $\mathbf{y}$, for various values of forcing parameter $F$, assuming that model was integrated forward from the true initial point. Only states $x_{1}, x_{4}, x_{7}, x_{10}$ were measured. The global minimum of $C(F)$ (dotted line; $F=8)$, resides in an extremely narrow basin of attraction within a highly nonconvex cost surface. B: Same as A, but for controlled dynamics $(u=4$ and $u=15$ for middle and right plots, respectively). Higher controls provide a far smoother cost function around the global minimum. C: 2D projection of the high-dimensional DSPE cost surface, along $F$ in one dimension and the line $U_{l}\left(t_{n}\right) \equiv u, \forall l, n$ in the other. Here, $x_{d}\left(t_{n}\right)$ were fixed at the values generated by integrating the model forward from its true initial state for the given $F$. D: Estimated trajectories of $x_{8}$, using uncontrolled least squares (top; green) and DSPE (bottom; red). Black line: true trajectory. Shaded regions: SD of estimate across 100 random initializations of the optimization. E: Histogram of parameter estimates across 100 initializations of the optimization, for least squares (green) and DSPE (red). 
where, in contrast to direct nudging, the control terms are now time-dependent, $\mathbf{U} \rightarrow \mathbf{U}\left(t_{n}\right)$. In this dynamical state and parameter estimation (DSPE) framework, the dynamical states $\mathbf{x}\left(t_{n}\right)$, control terms $\mathbf{U}\left(t_{n}\right)$, and model parameters $\boldsymbol{\Theta}$ are optimized simultaneously. With no control $\left(\mathbf{U}\left(t_{n}\right)=0\right)$, this optimization reduces to a naive constrained least squares matching of the model to the observations. The insight of DSPE is that the cost function becomes smoother for larger $\mathbf{U}(t)$, localizing the estimate to the vicinity of the global minimum, while the quadratic penalty on $\mathbf{U}(t)$ reduces the controls, refining the estimate to the true minimum of the uncontrolled dynamics. Thus, one can think of $\mathbf{U}(t)$ as opening up new degrees of freedom, allowing escape from local minima during the optimization procedure. When the optimization has terminated, the optimal $\mathbf{U}\left(t_{n}\right)$ - which are driven to small values due to the $\sim \mathbf{U}^{2}$ cost penalty - are discarded. In this sense, they are essentially an algorithmic device.

We illustrate the regularizing effects of the control parameters by plotting a $2 \mathrm{D}$ projection of the DSPE cost surface, along $F$ and along a 1D projection in the space of $\mathbf{U}\left(t_{n}\right)$ (Fig $\left.2 \mathrm{C}\right)$ : the surface has a broad minimum for larger control strength, and a complex, rugged surface as the control strength becomes weaker. To illustrate the performance of the DSPE algorithm, we compare it against a naive constrained least squares optimization without controlled dynamics, again using the 10D Lorenz96 system. Across 100 random initializations, both least squares and DSPE produced comparably accurate estimates of the state variables, with DSPE being somewhat more accurate near the boundaries of the time window (Fig 2D). The differences in the estimate of the forcing parameter $F$, however, were more striking. For nearly all initializations, DSPE estimated $F$ within $1 \%$ of its true value, while the distribution of parameters estimated by least squares peaked at the true value but were far more dispersed (Fig $2 \mathrm{E})$.

\section{Optimally-controlled dynamical parameter inference}

We now present our main results. We have seen that DSPE built naturally on nudging synchronization, enforcing controlled dynamics as constraints in a global optimization over states, parameters, and time-dependent controls. Here, we suggest that the control variables, rather than acting as an algorithmic device to regularize the optimization procedure, could be instead specified in a more principled manner, by viewing DSPE as the foundation for an optimal control problem. In this framework, the optimal estimate can be derived using the Pontryagin minimum principle. Using the DSPE cost function Eq 6, expressed in continuous time,

$$
C=\int_{0}^{T} d t \mathcal{L}(\mathbf{x}, \mathbf{U})=\int_{0}^{T} d t\left[\frac{1}{2}\|\mathbf{H} \mathbf{x}-\mathbf{y}\|^{2}+\|\mathbf{U}\|^{2}\right],
$$

and the controlled dynamics Eq 3 , we apply the minimum principle in the usual way (Methods for derivation) to obtain an expression for the optimal control (assuming $\mathbf{U}$ is diagonal for readability; this can be relaxed trivially):

$$
U_{l l}=-p_{l}[\mathbf{y}-\mathbf{H x}]_{l}, \quad\left(\text { for } x_{l} \text { observed }\right),
$$

This control can be used to derive the dynamics obeyed by the optimal estimate and the conjugate momenta $p_{d}$ in time:

$$
\begin{array}{cc}
\dot{x}_{d}^{*} & =f_{d}\left(\mathbf{x}^{*}, \boldsymbol{\Theta}\right)-p_{d}^{*}\left(\left[\mathbf{y}-\mathbf{H x}^{*}\right]_{d}\right)^{2} \\
\dot{p}_{d}^{*}=-\frac{\partial \mathbf{f}\left(\mathbf{x}^{*}, \boldsymbol{\Theta}\right)}{\partial x_{d}^{*}} \cdot \mathbf{p}^{*}+\left[\mathbf{H}^{T}\left(\mathbf{y}-\mathbf{H x}^{*}\right)\right]_{d}\left(1-\left(p_{d}^{*}\right)^{2}\right)
\end{array}
$$

where the asterisk refers to the fact that the integral curves of these equations represent locally optimal trajectories. Since this dynamical system describes the evolution of the optimal estimate, we call it the estimation dynamics. Note the the estimation dynamics 
form a boundary value problem rather than an initial value problem [49]: $x_{d}$ in the null-space of $\mathbf{H}$ are unobserved, so their values at $t=0$ are unspecified. A wealth of studies have been devoted to solving control systems of this class, using polynomial approximations - so-called collocation methods - or instead using shooting methods, which solves the initial value problem perturbatively until the boundary conditions are matched [50]. Our problem has the added complication that with unknown parameters, the dynamics could not be integrated forward in either case. Instead, we propose an algorithm for simultaneously estimating $\mathbf{x}, \mathbf{p}$, and $\boldsymbol{\Theta}$ that leverages some desirable aspects of the original cost function $\mathcal{L}(\mathbf{x}, \mathbf{U})$ in a practical implementation. We first use the optimal control condition Eq 9 to express $\mathcal{L}, \mathrm{Eq} 8$, in $\{\mathbf{x}, \mathbf{p}\}$ space:

$$
\tilde{\mathcal{L}}(\mathbf{x}, \mathbf{p})=\sum_{l} \frac{1}{2}\left[\left([\mathbf{y}-\mathbf{H} \mathbf{x}]_{l}\right)^{2}\left(1+\left(p_{l}\right)^{2}\right)\right]
$$

This is the original cost function to be optimized, now written in $\mathbf{x}, \mathbf{p}$. Since the estimation dynamics Eqs. 10 fully define the optimal trajectory, $\tilde{\mathcal{L}}$ contains no new information [49]. Nevertheless, this function must be stationary along locally optimal trajectories. Further, it is convex in the space of observable $\mathbf{x}$ and associated $\mathbf{p}$, so its minimum is global in those directions, and highly degenerate in the unmeasured directions.

We could therefore use the solutions of $\min \tilde{\mathcal{L}}$ as a starting point for the more complicated task of satisfying the highly nonlinear estimation dynamics. Specifically, we could begin by optimizing $\tilde{\mathcal{L}}(\mathbf{x}, \mathbf{p})$, subject to a loose enforcement of Eqs. 10 . The resultant path and parameters would then be used as the initialization for a subsequent minimization of $\tilde{\mathcal{L}}(\mathbf{x}, \mathbf{p})$ subject to Eqs. 10 , now enforced to a tighter degree. We call this method "optimally-controlled dynamical state and parameter estimation" (OC-DSPE), and schematize it in Algorithm 1. The idea of OC-DSPE is that the enforcement of the nonlinear dynamics breaks the convexity in a gradual manner, allowing the global minimum to be systematically tracked - in much the same spirit as the control variable directions in the DSPE cost manifold (vertical direction in Fig 2 $\mathrm{C}$ ). This iterative procedure lies in a class of nonlinear techniques called homotopy continuation, where the solutions of one system are tracked by iterating from a simpler system with known solutions [41]. Incorporating this flavor of homotopy continuation into nonlinear estimation was the basis of a number of studies, in particular quasi-static variational assimilation [51] and variational annealing $7,3,32,34]$. The latter has been shown to be quite effective in tracking highly chaotic systems, as well as in highly under-observed neuron models with many unspecified nonlinear parameters. A more recent study illustrated benefits in nonlinear system estimation by extending variational annealing to Hamiltonian manifolds [4], in much the same spirit as Algorithm 1 .

\section{Numerical experiments of OC-DSPE with 10-dimensional Lorenz96 model}

We first apply OC-DSPE to the Lorenz96 system in 10 dimensions with unknown forcing parameter $F$, performing 100 optimizations with different initial guesses (Methods for implementation details). We first show illustrative plots of the state estimates, assuming $M=4$ states are observed. For the observed $\mathbf{x}_{1}$, the state estimate averaged over all 100 estimations is well-localized around the true trajectory ( Fig $3 \mathrm{~A}$; top plot). The unobserved state variable is markedly less accurate (Fig $3 \mathrm{~B}$; top plot), but does approximate certain regions well (i.e. $t \sim 4-6$ ), suggesting that the chaotic instabilities are less pronounced in those regions around the attractor. Of course, since the trajectories depend on the parameters, the accuracy of the states is often correlated with that of the parameters. If we plot the state estimates for which the forcing parameter is estimated close to its true value, $\left|\hat{F}-F_{\text {true }}\right|<0.05$, we find that the states 


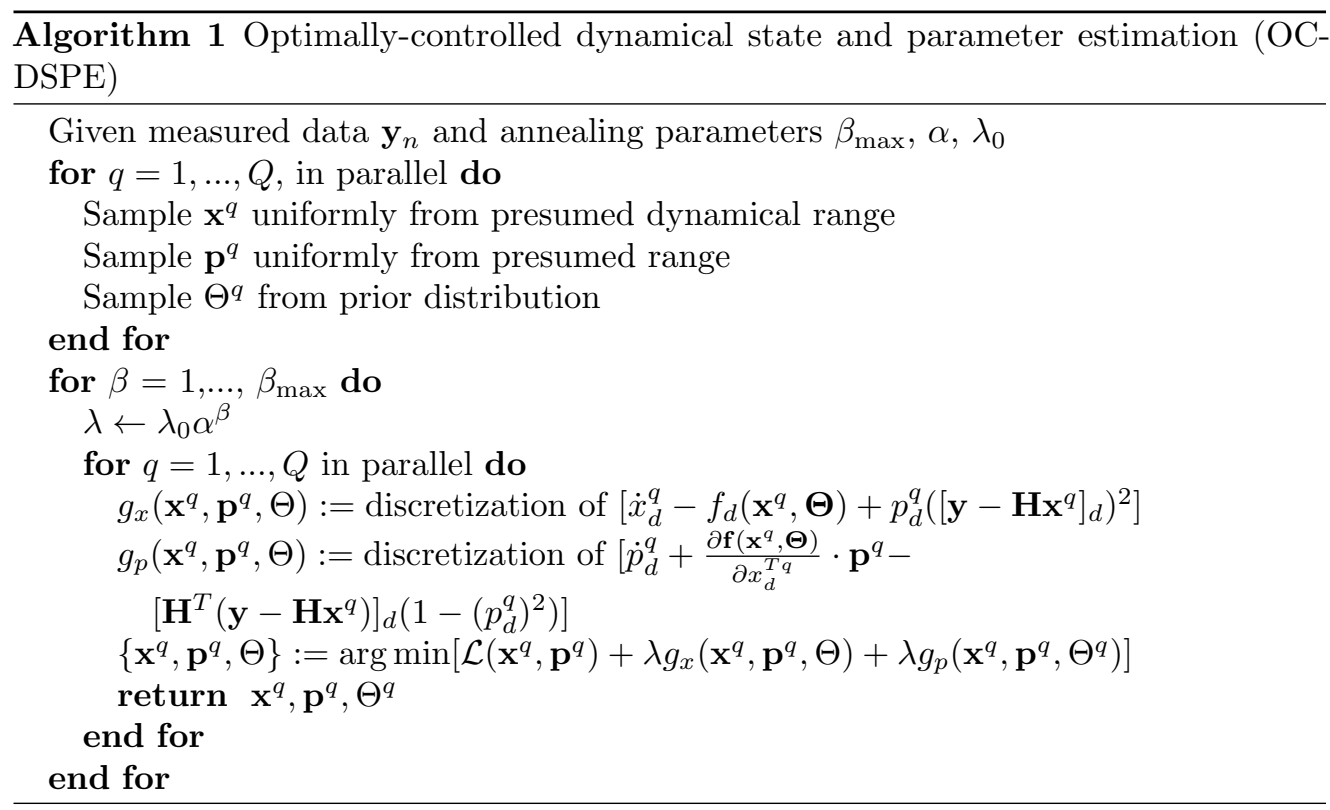

are nearly indistinguishable from the true trajectories, beyond an initial synchronization region, $t>\sim 1$ (Fig $3 \mathrm{~A}-3 \mathrm{~B}$; bottom). This initial synchronization region is not surprising. The optimal control quickly nudges the estimate toward the observed data, even if the state at the beginning of the estimation window $t=0$ is highly inaccurate. Once synchronized, the optimal control maintains the estimate near the true trajectory at minimal control cost $\mathbf{U}^{2}$.

Next, we focus on the accuracy of parameter estimates and compare i) OC-DSPE, ii) DSPE, and iii) constrained least squares (see Methods for details of the implementation of each method). We repeated the 100 estimations for $Z=100$ distinct estimation windows around the Lorenz attractor to get a better representation of the estimation in regions where the chaotic instabilities are both strong or weak. We first plot the distribution of estimated parameters when $M=5$ states $\left(x_{d} ; d\right.$ odd) are observed. For $M=5$, the estimated parameters are highly localized around the true value for all techniques (Fig $3 \mathrm{C}$ ). The advantage of OC-DSPE becomes more apparent with fewer measured states. In particular, with $M=2$ observed states, the estimates for all parameters are substantially more dispersed (Fig $3 \mathrm{D}$ ). Only OC-DSPE, however, is centered near on the true value; DSPE in particular is highly dispersed giving erroneous parameter estimates as low as 0 . This illustrates that OC-DSPE, while computationally more demanding (the state space is doubled and the equations require more derivatives), can produce substantially more accurate parameter estimates in very sparsely observed chaotic systems.

To further quantify the robustness of the estimation procedure, we calculated the estimated parameter as a function of the error between observations and estimates, $E=\sqrt{\sum_{n}\left\|\mathbf{H} \hat{\mathbf{x}}\left(t_{n}\right)-\mathbf{y}\left(t_{n}\right)\right\|^{2}}(\mathrm{Fig} 3 \mathrm{E})$. Here, we chose, for each of the $Z$ estimation windows, the optimal parameter estimate among the 100 initializations. This gives a "best-case" estimate for each dataset, and quantifies how this optimal parameter estimate depends on the errors in the dynamical states. Though all 3 algorithms produce accurate $\hat{F}$ when the estimation error is minimal, OC-DSPE is far more tightly centered on the true parameter value even as the state error increases. This indicates that for OC-DSPE i) the optimal parameter estimate could be found more reliably with less initializations and ii) the optimal estimate can be identified with more reliably in practice, where the only error metric available is $E$. 
$\mathbf{A}$

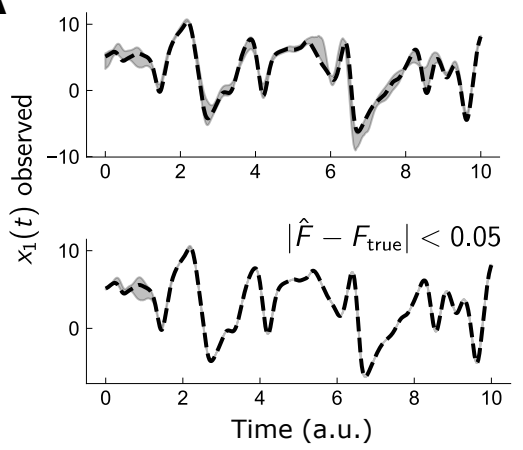

B

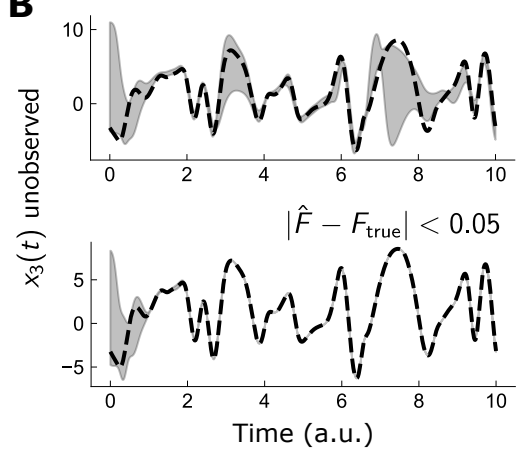

C

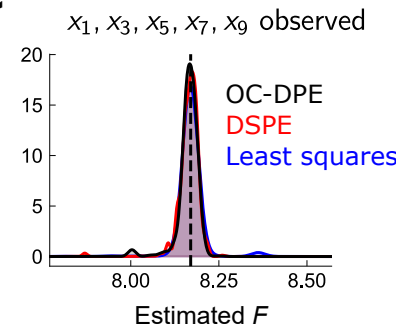

D

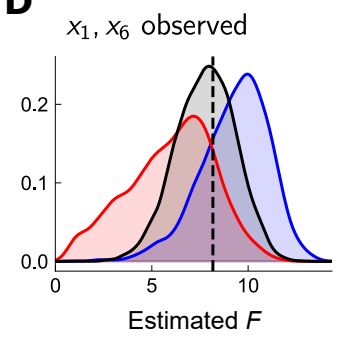

$\mathbf{E}$

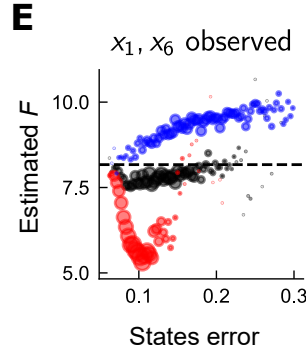

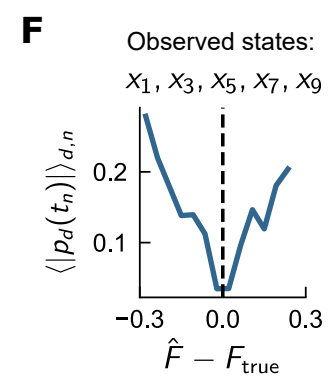
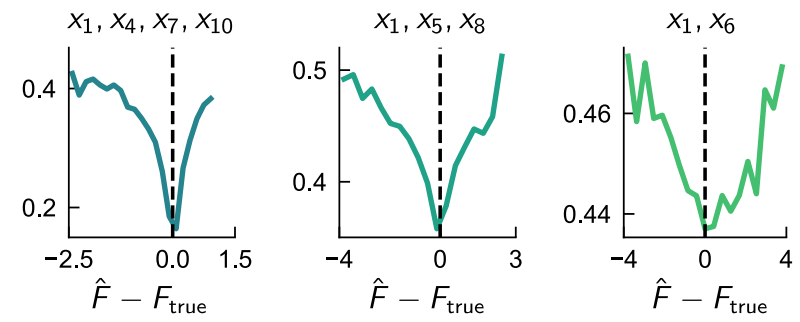

Fig 3. OC-DSPE exhibits enhanced parameter estimation accuracy in sparsely-observed chaotic models. Illustration of parameter estimation in chaotic Lorenz96 system using optimally-controlled dynamical state and parameter estimation (OC-DSPE). A: Illustrative estimate of observed variable $x_{1}(t)$ when 4 states are observed, for 100 estimations randomly initialized (top), and for only those estimations in which the parameter was estimated to high accuracy (bottom; $|\hat{F}-F|<0.05$ ). Grey: standard deviation of all estimates; Dotted: true state. B: Same as A, for the unobserved variable $x_{3}(t)$. C: Histogram of parameter estimates over 100 initializations, for 100 datasets initialized throughout the 10D Lorenz96 chaotic attractor, for 5 observed variables and using 3 different estimation routines (least squares, the original DSPE algorithm, and OC-DSPE). Parameter estimates are tightly clustered around the true value of 8.17. D: Same as C, for only 2 observed variables. Estimated $\hat{F}$ is more widely dispersed, but centered on the true value only for OC-DSPE. E: Estimated parameter $\hat{F}$ versus error in observed states $x$ averaged over space and time. For low state error, estimated parameters are close to the true value for all 3 estimation routines. Tor larger state errors, proximity to the true value occurs only in OC-DSPE, indicating a larger degree of robustness in estimation accuracy. F: Dependence of $\left|p_{d}\left(t_{n}\right)\right|$, averaged over dimension $i$ and time $n$, on error in parameter estimate, $\hat{F}-F$, for 5 (leftmost plot), 4,3 , and 2 (rightmost plot) observed states. In all cases, the minimum average $|p|$ occurs at very close to the true $F$, indicating that the values of the conjugate momenta can act as a proxy for identifying the optimal parameter estimate. 
Finally, we consider the role of the auxiliary momenta variables $p$ in OC-DSPE, a feature absent in direct optimization schemes. In the control-theoretic sense, $p$ represent the marginal cost of violating the constraints given by the system dynamics (Eq 7). Regions in which $p$ are appreciable correspond to regions in which the state estimate could easily deviate from the controlled dynamics, suggesting a lack of estimation accuracy (Fig $3 \mathrm{E}$ ). Do deviations in $p$ also have some bearing on the reliability in the parameter estimate? In other words, is there a correlation between some statistic of $p$ and the estimation errors in $F$ ? Indeed, plotting the magnitude of $p$ averaged over time and dimension as a function of $\hat{F}-F_{\text {true }}$ shows a prominent dip at zero parameter estimate error (Fig $3 \mathrm{~F}$ ). Thouhg this dip is more prominent for larger observability (leftmost plot in Fig $3 \mathrm{~F}$ )), the dip is still present even for very sparse observability (rightmost plot in Fig $3 \mathrm{~F}$ )). This indicates that the statistics of such artificial "conjugate" variables can provide a further consistency check on the accuracy of the estimation.

\section{Numerical experiments with the Morris-Lecar model}

Our primary system of interest is a biophysically realistic neuron modeled on the Hodgkin-Huxley framework. We use the Morris Lecar (ML) system 1], a reduced 2-state variable spiking neuron model in which the gating variable $w(t)$ drives changes in the neuron membrane voltage $V(t)$, and which has 12 static parameters (Methods). We first use OC-DSPE to estimate only the linear conductances $g_{i}$, which dictate the regimes of neuron excitability; we hold the other parameters fixed at their correct values. Observations $\mathbf{y}(t)=\left\{V_{\mathrm{obs}}(t)\right\}$ are generated by adding uncorrelated Gaussian noise $\sim \mathcal{N}(0, \sigma)$ to the ground truth voltage $V_{\text {true }}(t)$, where we investigate $\sigma=2 \mathrm{mV}$ and $\sigma=10 \mathrm{mV}$. The stimulating current is held at a constant value (Fig 4A). To illustrate the robustness of the algorithm to parameter uncertainty, we let the optimization bounds on the unknown conductance parameters span 2 orders of magnitude, from 0 to 200. To cross-validate our predictions, we forward integrate the dynamics from the final time estimate state $\hat{V}(T), \hat{w}(T)$ using the estimated parameters $\hat{g}_{i}$ but a pseudo-noisy current spanning a range of input currents (Fig 4A). We use $Q=25$ initializations in Alg. 1 .

For small measurement noise, $\sigma=2$, most of the $Q$ runs give highly accurate estimates of both the states $V(t)$ (Fig $4 \mathrm{~B}-\mathrm{C})$ and parameters $g_{i}$ (Fig $\left.4 \mathrm{D}\right)$. For the poorest fit, $g_{i}$ is sufficiently inaccurate such that spike events are occasionally missed. For higher measurement noise, $\sigma=10 \mathrm{mV}$, about $10 \%$ of the dynamic range, many predictions have degraded as expected, producing shifted or missed spike times (Fig $4 \mathrm{E}$ ). Still, the optimal prediction and estimated parameters are highly accurate among these 25 initializations (Fig $4 \mathrm{E})$.

As a more realistic numerical experiment, we next estimated of all 11 model parameters governing ion channel kinetics (we omit the capacitance $C$, which is typically ascertained from neuron size). All parameters are again bounded liberally, over 2 orders of magnitude. For low measurement noise, the model parameters, including those entering the model equations nonlinearly, are estimated to high precision - this is borne out in the accuracy of the forward predictions (Fig 5A-B). Still, as before, spikes can be missed and/or slightly shifted (Fig $5 \mathrm{C})$ ).

As noted in a number of prior studies [18,31], the accuracy of the estimation is inherently limited by the richness of the driving current. If, for example, the stimulating current were $0 \mathrm{pA}$ - below the spiking threshold - the estimated conductances would be degenerate: the fixed point of $\dot{V}$ would be unchanged by appropriate rescalings of $g_{i}$. Difficulties in estimating the kinetic parameters such as $\beta_{i}, \gamma_{i}$ would also arise since there are no observations when the neuron is spiking. In the case of a single step, as in Fig 4A and Fig 5A, the volume of phase space in $V(t), w(t)$ occupied by the system as again vanishingly small, covering only the closed curve along the spiking limit cycle. 


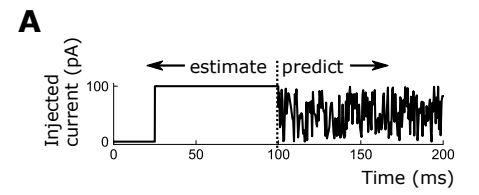

B

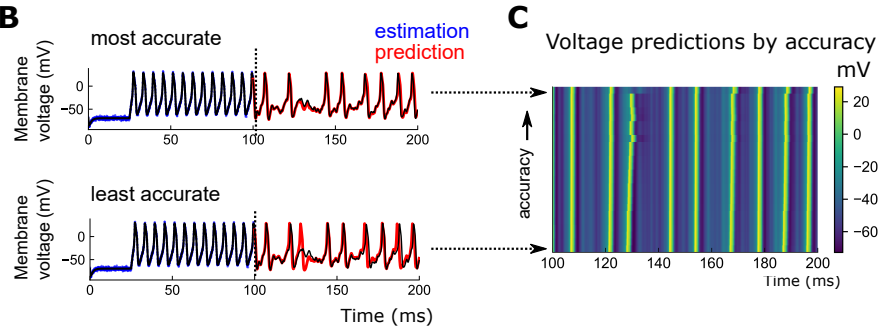

E

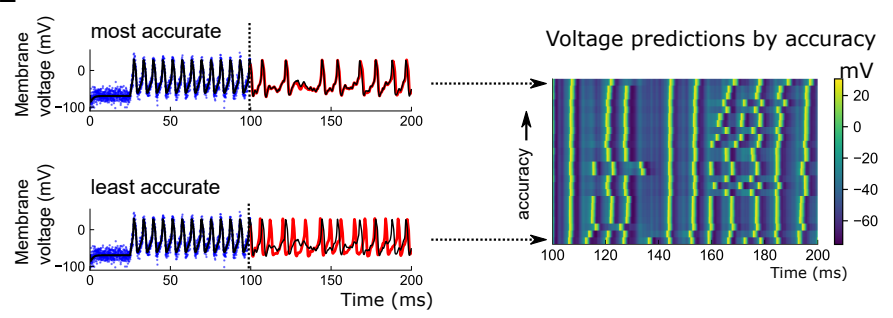

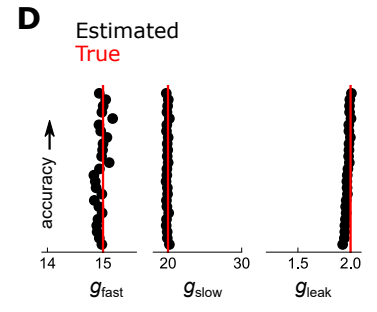

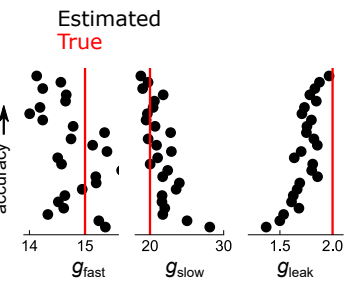

Fig 4. Estimation of states and linear conductances in Morris-Lecar model A: Stimulating current used to estimate parameters and compare forward predictions. B: Most accurate (top) and least accurate (bottom) of 25 estimations using OC-DSPE, $\sigma=2 \mathrm{mV}$. Black: true voltage; blue: noisy observations; red: forward prediction using estimated parameters. C: Heatmap of voltage over time for all 25 runs, with the run giving lowest (highest) prediction error on top (bottom). D: Estimated conductances corresponding to state estimations in C. E: Analogue of B-D, for higher measurement noise $\sigma=10 \mathrm{mV}$. 
A

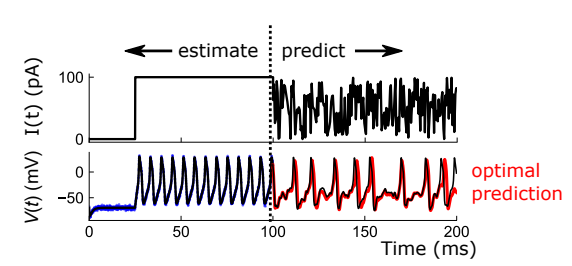

B

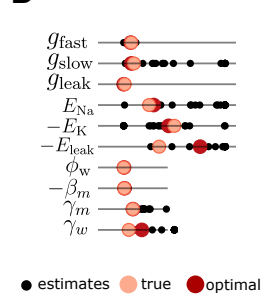

C

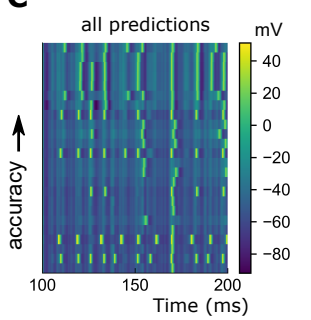

G
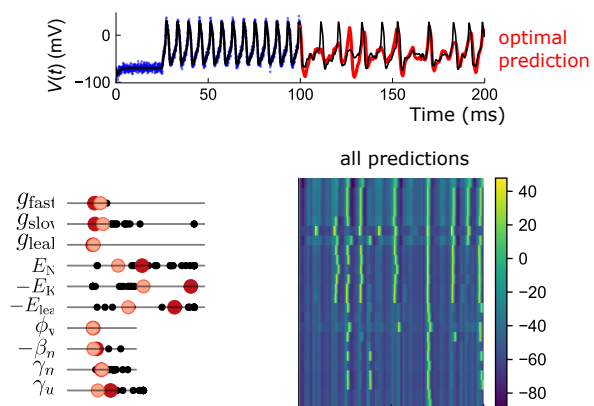

all predictions

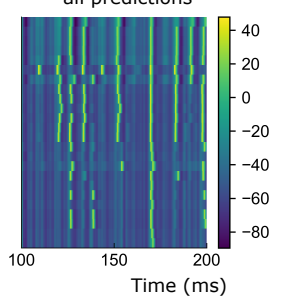

D

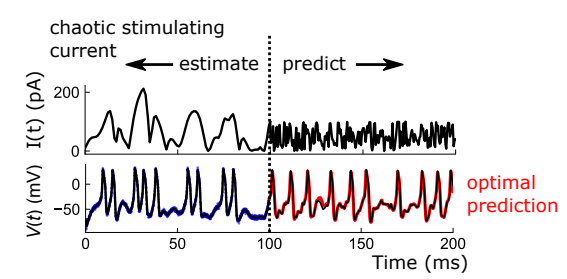

$\mathbf{E}$

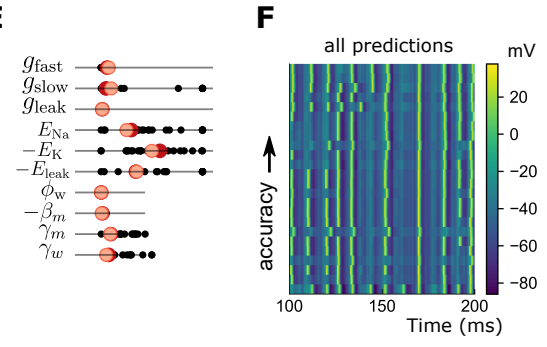

H
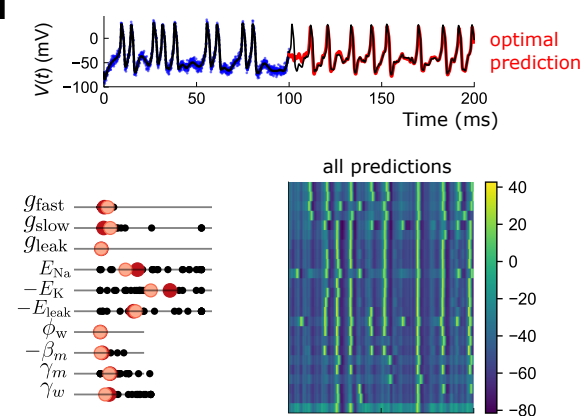

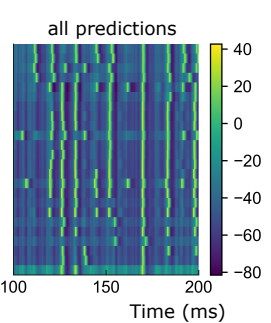

Fig 5. Estimation of linear and nonlinear parameters in Morris-Lecar model. A: (top) Stimulating current used for estimation and prediction. (bottom) Black: true voltage trace; blue: noisy observations; red: predicted voltage trace with highest accuracy. B: Estimated parameters for all 25 runs. Black: individual 25 estimates of each parameter; pink: true value of parameter; red: parameter estimate corresponding to lowest prediction error. C: Predicted voltage traces from parameters, from highest accuracy (top) to lowest accuracy (bottom), for all 25 runs. D-F; same as A-C but now using a complex stimulating current. Prediction accuracy is markedly more reliable. G-H: Same as A-F, for higher noise $(\sigma=10 \mathrm{mV})$, for step stimulating current $(\mathrm{G})$ and complex stimulating current $(\mathrm{H})$. In the presence of higher noise, optimal state and parameter estimates are nonetheless very accurate for complex stimulating currents $(\mathrm{H})$. 
Instead, valuable information toward the parameter estimates could be gained by pushing the system to less-visited regions of phase space. One option is to use a staircase signal consisting of many step currents 31. Alternatively, by using a dynamic current, we could persistently modulate the system between different limit cycle manifolds. Since the shape of these manifolds differs by input current, and since steady state is never achieved, the system would cover a larger swath of the phase space through transient motion 18]. This richer sampling of the phase space would increasingly enhance our estimate of $g_{i}$.

We therefore repeated the experiments using a stimulating current proportional to the $x$ component of the 10D Lorenz96 system (we took the absolute value so all currents were positive, and linearly scaled the time axis) (Fig 5D). In the optimally predicted $\hat{V}(t)$, the shifts in spike times are now absent, and the dropped spike near $t \sim 200$ is recovered (Fig 5D). The estimated parameters are also more precise, producing markedly more robust predictions (Fig $5 \mathrm{E}-\mathrm{F})$. Finally, we repeat the experiments for the higher measurement noise $\sigma=10 \mathrm{mV}$. The optimal prediction for the step current are of moderate accuracy: many spikes are reconstructed but many are dropped (Fig 5 G). For the chaotic driving current, spike times in the optimal prediction match very well against the data $(\operatorname{Fig} 5 \mathrm{H})$.

\section{Discussion}

Mathematical models for biological neuron and circuit models are informed intimately by neurobiology. Voltage dynamics in the Hodgkin-Huxley model, for example, obey classical electrodynamic equations for variable capacitors, reflecting the assumption of cell membranes as insulating barriers separating charged species. But while biological considerations may strongly constrain the model description, the many parameters in these models are unspecified. In fact, it is precisely the variability of these parameters that accounts for the vast diversity in system response, and ultimately dictates how these neural systems process external stimuli.

Here, we propose an optimization-based method for extracting parameters of biophysical neural models from noisy and incomplete measurements. Our approach is to cast the inference problem into an optimal control framework, resulting in a system of coupled ODEs for the dynamics obeyed by the optimal estimate. We propose a constrained optimization algorithm to iteratively solve this otherwise unstable system, and illustrate the accurate estimation of parameters in undersampled chaotic and neural models of interest.

Several studies in recent years have developed direct optimization-based approaches for state and parameter estimation in neural systems $6,6,32,34,40,49,52,53$. . Directly optimizing high-dimensional distributions may seem computationally prohibitive, since the search space is large - equal to the number of dynamical states times the number of measurement times, plus the number of constant parameters. But optimization in state-space models benefits from the highly sparse structure of the Hessian matrix 6], making these methods amenable to fast linear algebraic routines. While our approach does not directly optimize the usual posterior distribution appearing in the Bayesian setting [5.6], the Hessian matrices required by Newton-type minimizers have a similarly sparse structure, so the computational benefits remain.

The integral equations describing the evolution of the optimal estimate, Eq 10 , pose two difficulties: they are persistently unstable since they comprise a Hamiltonian system, and they are not directly integrable anyways, since the parameters are not specified. We address these two issues simultaneously by imposing these dynamics as constraints on a related, tractable cost function, and enforcing them iteratively as a penalty term. This is similar to a recent method identifying state space inference with 
Hamiltonian systems [49], and shares overlaps with a related homotopy approach for parameter estimation in nonlinear systems [42]. These approaches and ours adopt the viewpoint that, rather than i) recursively filtering with linearized dynamics or ii) attempting to represent high-dimensional distributions with particle estimates, the burden of nonlinearity can be shifted to the cost function, provided that nonconvexity can be introduced in a systematic way. Globally optimizing over these variables is, of course, only possible for data that is analyzed offline, as in calcium imaging or electrophysiological measurements.

A recent work 31 has explored how suboptimal parameter estimates can be "nudged" out of local minima by adding artificial measurement noise. That technique produced highly accurate estimations of 41 parameters in a conductance-based neuron model, though compared to our results here, the measurement noise was 10 times smaller. Still, one could envision incorporating such "noise-regulation" in our method here to suppress the impact of local minima.

Our emphasis is on estimation accuracy, rather than computational cost. Indeed, both doubling the state space to a Hamiltonian system and imposing constraints increases compute time compared to, for example, the variational annealing method [32,33], or directly optimizing the least squares error subject to hard model constraints. Extending this framework to larger systems would require a careful consideration of the tradeoffs between accuracy and speed. Still, the accurate estimation of a large number of nonlinear parameters suggests that our method, combined with improved optimization routines, may be relevant for larger neural systems.

We have focused here on estimation of parameters in single neuron models, the obvious extension being to the inference of biophysical neuron parameters and connection weights in neural networks. Inference in neural networks will demand a different modeling approach, since highly-resolved voltage waveforms from multiple neurons in vivo are inaccessible, given current technologies. It is unlikely that detailed conductance-based models would be resolvable, and one might opt instead for reduced phenomenological models [1 26] sharing the desired dynamical features, but containing richer dynamics than simple perceptrons. Our methodology is most relevant to data taken in vitro, where currents can be externally controlled. Fruitful directions for future research would carefully consider these limitations, deciding how best to adapt this and other model-based procedures for brain-wide calcium imaging data, as has been explored in recent studies [28].

\section{Materials and methods}

\section{DSPE cost function generation}

To generate the data used in Fig $2 \mathrm{~A}$, we numerically integrated the Lorenz96 system in 10 dimensions using the scipy.odeint routine in Python, which runs the FORTRAN solver LSODE. The integration was performed over 501 timepoints from $t_{0}=0$ to $t_{N}=8$ at a step of $d t=0.016$. Observations $y_{i}$ were generated by adding uncorrelated Gaussian noise $\sim \mathcal{N}(0,1)$ to the 4 observed states $x_{1}, x_{4}, x_{7}, x_{10}$. The states used in Fig $2 \mathrm{~B}$ were generated similarly, but using the controlled dynamics $\mathrm{Eq} 3$ with $U_{11}=U_{44}=U_{77}=U_{10,10}=u$.

For the cost surface representation in Fig $22 \mathrm{C}$, we used measurement noise $\sim \mathcal{N}(0,0.1)$; the observed states were as above. The full cost surface is technically 5 -dimensional - the $4 U_{l l}$ plus the forcing $F$. To plot a visualizable cross section of this surface, we set all $U_{l l}$ to be the same - this is equivalent to projecting the surface along that line. 


\section{Neuron model}

The Morris-Lecar ODE dynamics are given by:

$$
\begin{gathered}
C \dot{V}=-g_{\text {fast }} m_{\infty}(V)\left(V-E_{\mathrm{Na}}\right)- \\
\quad g_{\text {slow }} w\left(V-E_{\mathrm{K}}\right)-g_{\text {leak }}\left(V-E_{\text {leak }}\right)+I_{\text {stim }} \\
=\phi_{w} \frac{w_{\infty}(V)-w}{\tau_{w}(V)}
\end{gathered}
$$

where

$$
\begin{array}{cc}
w_{\infty}(V) & =0.5\left(1+\tanh \left(\frac{V-\beta_{\mathrm{w}}}{\gamma_{\mathrm{w}}}\right)\right) \\
m_{\infty}(V) & =0.5\left(1+\tanh \left(\frac{V-\beta_{\mathrm{m}}}{\gamma_{\mathrm{m}}}\right)\right) \\
\tau_{\mathrm{w}}(V) & =\left(\cosh \frac{V-\beta_{\mathrm{w}}}{2 \gamma_{\mathrm{w}}}\right)^{-1}
\end{array}
$$

The ground truth parameters were set at as $C=2.5, g_{\text {fast }}=20, g_{\text {slow }}=15, g_{\text {leak }}=2$, $E_{\mathrm{Na}}=50, E_{\mathrm{K}}=-100, E_{\text {leak }}=-70, \phi_{\mathrm{w}}=0.12, \beta_{\mathrm{w}}=0, \beta_{\mathrm{m}}=-1.2, \gamma_{\mathrm{m}}=18, \gamma_{w}=10$. Data used for state and parameter estimation was generated by integrating these dynamics with a prescribed stimulating current $I_{\text {stim }}$, over a window of $[0,100] \mathrm{ms}$ at a timestep of $0.05 \mathrm{~ms}$. For the step stimulus (Fig 4 and Fig $5 \mathrm{~A}-\mathrm{C}, \mathrm{G}$ ), the current was set to $100 \mathrm{pA}$. For the chaotic stimulating current (Fig $5 \mathrm{D}-\mathrm{F}, \mathrm{H})$ ), we used the absolute value of the output of 1 variable of the 10D Lorenz96 system, scaled in time by a factor of 15 and in value by a factor of 20 .

\section{Constrained optimization}

The estimated states and parameters were all found with constrained optimization using the limited-memory Broyden-Fletcher-Goldfarb-Shanno algorithm with constraints (L-BFGS-B), implemented in Python with the package scipy.optimize.minimize. These optimizations are high-dimensional. The search space in DSPE is

$N D+N L+P$-dimensional, where $N$ is the number of timepoints, $D$ is the dimension of the dynamical system, $L$ is the number of measurements, and $P$ is the number of parameters. The $N L$ term accounts for the control variables $U_{l l}\left(t_{n}\right)$, one for each observed variable at each timepoint. For least squares, the search space is $N D+P$ dimensional, and for OC-DSPE, the search space is $2 N D+P$-dimensional to account for the momenta variables. Bounds must be supplied on all state and parameter variables; for the Lorenz96 system, the states were bounded in $[-15,15], F$ was bounded in $[1,20]$, and $U_{l l}\left(t_{n}\right)$ were bounded in $[0,100]$. For the Morris Lecar system, $V$ was bounded in $[-100,100]$, the gating variable $w$ in $[0,1]$, and the in $[0.01,200]$. For OC-DSPE, the momenta $p$ must also be bounded; these were set at $[-100,100]$.

Equality constraints were required for all 3 methods - least squares, DSPE, and OC-DSPE. These constraints enforced either the raw, uncontrolled system dynamics (least squares; Eq 1), the controlled system dynamics (DSPE; Eq 7), or the estimation dynamics (OC-DSPE; Eq 10). The constraints were enforced iteratively with a penalty method. Specifically, for all 3 routines, the following function was minimized.

$$
C(\beta)=C(\cdot)+2^{\beta} \sum_{i, n} R_{d} g_{i, n}^{2}(\cdot)
$$

where $C(\cdot)$ is the cost function and $g_{i, n}(\cdot)$ is a discretization of the $i$ th constraint equation at timepoint $t_{n}$, and $\beta$ was iteratively stepped up from 0 to 24 . Specifically, $C(\beta=0)$ was minimized with a randomly chosen initialization of all states and parameters within their bounds; the result of this was used as the initial guess for the minimization of $C(\beta=1)$, etc. The estimate at $\beta=24$ was used taken to be the 
optimal estimate. $C(\cdot)$ for DSPE was Eq 6 , for least squares was Eq 6 absent the $\mathbf{U}$ terms, and for OC-DSPE was Eq11. The constraints were discretized using Hermite-Simpson quadrature, which results in $N$ constraint equations for each $i . R_{i}$ is a constant factor that allows the individual constraints to scale respectively with the dynamic range of that particular variable; this normalizes the contributions of different variables to the penalty term. For the Lorenz system $R_{i}=1 \mathrm{e}-4, \forall i$; for the neuron model, $R_{V}=1 \mathrm{e}-4, R_{w}=1, R_{p_{V}}=1, R_{p_{w}}=1$. Note that the least squares method with iteratively enforced constraints is equivalent to the variational annealing algorithm proposed previously $18,32,34,49,53$. These estimations were done many times in parallel (100 for Lorenz96 system; 25 for the neuron model) with different initial guesses for the parameters and states at $\beta=0$.

For the Morris-Lecar neuron model, once the optimal state and parameter estimates are determined, they were used to generate predictions in the interval $[100,200]$ by integrating forward the dynamics with the final state estimate $\mathbf{x}\left(t_{N}\right)$ using the estimated parameter values.

All optimization routines required first derivatives of the cost function and the constraints; we obtained these in a few lines of code by using the automatic differentiation package PyAdolc.

\section{OC-DSPE estimation dynamics}

The optimal control conditions were derived using the Pontryagin Minimum Principle 43. In this formulation, one must prescribe the cost function along with the system dynamics; each of these may depend on the states, parameters, and controls. Our cost function is the DPE cost function Eq 6 (in continuous time) - a least squares matching of observations and states, plus a quadratic control penalty (restatement of $\mathrm{Eq} 8$ from the main text):

$$
C=\int_{0}^{T} d t \mathcal{L}(\mathbf{x}, \mathbf{U})=\int_{0}^{T} d t\left[\frac{1}{2}\|\mathbf{H} \mathbf{x}-\mathbf{y}\|^{2}+\|\mathbf{U}\|^{2}\right]
$$

where $\mathcal{L}$ is the system "Lagrangian." The control optima are derived from the corresponding "Hamiltonian" function which is defined from the Lagrangian via:

$$
\mathcal{H}(\mathbf{x}, \mathbf{U}, \mathbf{p})=\mathcal{L}(\mathbf{x}, \mathbf{U})+\mathbf{p} \cdot \mathbf{f}(\mathbf{x}, \mathbf{U}, \mathbf{\Theta})
$$

where $\mathbf{p}$ is conjugate momentum variable. Note that $\mathbf{x}, \mathbf{U}$, and $\mathbf{p}$ are all time-dependent, with the time-dependence suppressed for readability. The Pontryagin Minimum Principle states that then the optimal control $\mathbf{U}_{\text {opt }}$ along the integral curves of this system must satisfy:

$$
\mathbf{U}_{\mathrm{opt}}=\underset{\mathbf{U}}{\arg \min } \mathcal{H}(\mathbf{x}, \mathbf{U}, \mathbf{p}) \text {. }
$$

If the optimal control exists in the interior of the space of admissable controls, then the minimum of $\mathrm{Eq} 16$ can be found from the stationary points of $\mathcal{H}$ with respect to $\mathbf{U}$ :

$$
0=\frac{\partial \mathcal{H}}{\partial \mathbf{U}}
$$

which, for the nonzero diagonal elements of $\mathbf{U}$ satisfy

$$
0=\frac{\partial H}{\partial U_{l l}}=U_{l l}+p_{l}[\mathbf{y}-\mathbf{H x}]_{l}
$$

In addition to the condition on the stationarity of $\mathcal{H}$, the system dynamics must satisfy Hamilton's equations, one of which reproduces the state space dynamics $\dot{\mathbf{x}}$, while the 
second gives the dynamics for the conjugate momenta (writing out the indices explicitly):

$$
\begin{gathered}
\dot{x}_{d}=\frac{\partial \mathcal{H}}{\partial p_{d}}=f_{\text {cont }, d}(\mathbf{x}, \mathbf{U}, \boldsymbol{\Theta}) \\
\dot{p}_{d}=-\frac{\partial \mathcal{H}}{\partial x_{d}}=-\frac{\partial \mathcal{L}}{\partial x_{d}}-\frac{\partial \mathbf{f}_{\mathrm{cont}}(\mathbf{x}, \mathbf{U}, \boldsymbol{\Theta})}{\partial x_{d}} \cdot \mathbf{p}
\end{gathered}
$$

Writing out the derivatives of $\mathcal{L}$, and expanding out $f_{\text {cont }}$, this gives the following:

$$
\begin{array}{cc}
\dot{x}_{d} & =f_{d}(\mathbf{x}, \boldsymbol{\Theta})+[\mathbf{U} \cdot(\mathbf{y}-\mathbf{H x})]_{d} \\
\dot{p}_{d}=-\left[\mathbf{H}^{T}(\mathbf{H} \mathbf{x}-\mathbf{y})\right]_{d}+\left[\mathbf{H}^{T} \mathbf{U}^{T} \mathbf{p}\right]_{d}-\frac{\partial \mathbf{f}(\mathbf{x}, \mathbf{U}, \mathbf{\Theta})}{\partial x_{d}} \cdot \mathbf{p}
\end{array}
$$

The momenta equations $\dot{\mathbf{p}}$ provide dynamical constraints on the control variables $U_{a a}$, absent from nudging and DSPE. For observable states, the optimality condition Eq 18 can be inverted for the momenta $p_{d}$ (Eq. 9p. Applying this in Eq20 gives the complete set of optimally-controlled dynamics, in Hamiltonian coordinates $\{\mathbf{x}, \mathbf{p}\}, \mathrm{Eq} 10$.

\section{Acknowledgments}

We thank Paul Rozdeba for helpful discussions, and Viraaj Jayaram and Gustavo Madeira Santana for a careful reading of the manuscript. NK was supported by a postdoctoral fellowship through the Yale Swartz Foundation for Theoretical Neuroscience, by postdoctoral fellowship NIH F32MH118700, and by postdoctoral fellowship NIH K99DC019397.

\section{References}

1. Izhikevich EM. Dynamical Systems in Neuroscience: The Geometry of Excitability and Bursting. MIT Press; 2007.

2. Johnston D, Wu SMS. Foundations of Cellular Neurophysiology. MIT Press; 1995.

3. Dayan P, Abbott LF. Theoretical Neuroscience: Computational and Mathematical Modeling of Neural Systems. The MIT Press; 2005.

4. Hodgkin AL, Huxley AF. A quantitative description of membrane current and its application to conduction and excitation in nerve. The Journal of Physiology. $1952 ; 117: 500-544$.

5. Särkkä S. Bayesian Filtering and Smoothing. Cambridge University Press; 2013.

6. Paninski L, Ahmadian Y, Ferreira DG, Koyama S, Rad KR, Vide M, et al. A new look at state-space models for neural data. Journal of Computational Neuroscience. 2010;29:107-126. doi:10.1007/s10827-009-0179-x.

7. Kadakia N. The Dynamics of Nonlinear Inference. PHD thesis, ProQuest ID: Kadakia_ucsd_0033D_16631, UC San Diego; 2017.

8. Durstewitz D, Koppe G, Toutounji H. Computational models as statistical tools. Current Opinion in Behavioral Sciences. 2016;11:93-99. doi:https://doi.org/10.1016/j.cobeha.2016.07.004.

9. Moye MJ, Diekman C. Data Assimilation Methods for Neuronal State and Parameter Estimation. Journal of Mathematical Neuroscience. 2018;8. 
10. Wu W, Gao Y, Bienenstock E, Donoghue JP, Black MJ. Bayesian population decoding of motor cortical activity using a Kalman filter. Neural Computation. 2006;18:80-118.

11. Wu W, Kulkarni JE, Hatsopolous NG, Paninski L. Neural decoding of hand motion using a linear state-space model with hidden states. IEEE Transactions on Neural Systems and Rehabilitation Engineering. 2009;17:370-378.

12. Wei Y, Ullah G, Parekh R, Ziburkus J, Schiff SJ. Kalman filter tracking of intracellular neuronal voltage and current. In: 2011 50th IEEE Conference on Decision and Control and European Control Conference; 2011. p. 5844-5849.

13. Ullah G, Schiff SJ. Assimilating Seizure Dynamics. PLOS Computational Biology. 2010;6:1-12. doi:10.1371/journal.pcbi.1000776.

14. Paninski L, Vidne M, DePasquale B, Ferreira DG. Inferring synaptic inputs given a noisy voltage trace via sequential Monte Carlo methods. Journal of Computational Neuroscience. 2012;33:1-19. doi:10.1007/s10827-011-0371-7.

15. Box M, Jones MW, Whiteley N. A hidden Markov model for decoding and the analysis of replay in spike train. Journal of Computational Neuroscience. 2016;41:339-366. doi:10.1007/s10827-016-0621-9.

16. Brockwell A, Rojas A, Kass R. Recursive Bayesian decoding of motor cortical signals by particle filtering. Journal of Neurophysiology. 2004;91:1899-1907.

17. Meliza CD, Kostuk M, Huang H, Nogaret A, Margoliash D, Abarbanel HD. Estimating parameters and predicting membrane voltages with conductance-based neuron models. Biological Cybernetics. 2014;108:495-516.

18. Kadakia N, Armstrong E, Breen D, Morone U, Daou A, Margoliash D, et al. Nonlinear statistical data assimilation for HVCRA neurons in the avian song system. Biological Cybernetics. 2016;110:417-434.

19. Nogaret A, Meliza CD, Margoliash D, Abarbanel HDI. Automatic construction of predictive neuron models through large scale assimilation of electrophysiological data. Scientific Reports. 2016;6:32749.

20. Breen D, Shirman S, Armstrong E, Kadakia N, Abarbanel HDI. HVC interneuron properties from statistical data assimilation. arXiv. 2016;1608.04433.

21. Vogelstein JT, Watson BO, Parker AM, Yuste R, Jedynak B, Paninski L. Spike Inference from Calcium Imaging Using Sequential Monte Carlo Methods. Biophysical Journal. 2009;97:636-655. doi:10.1016/j.bpj.2008.08.005.

22. Kalman RE. A New Approach to Linear Filtering and Prediction Problems. Journal of Basic Engineering. 1960;82(Series D):35-45. doi:10.1115/1.3662552.

23. Julier SJ, Uhlmann JK. A new extension of the Kalman Filter. In: Proceedings of AeroSense: The 11th International Symposium on Aerospace/Defence Sensing, Simulation, and Controls; 1997.

24. Doucet A, Godsill S, Andrieu C. On sequential Monte Carlo sampling methods for Bayesian filtering. Statistics and Computing. 2000;10:197-208.

25. Kitagawa G. A self-organizing state-space model. Journal of the American Statistical Association. 1998;93:1203-1215. 
26. Huys QJM, Paninski L. Smoothing of, and parameter estimation from, noisy biophysical recordings. PLoS Computational Biology. 2009;5:e1000379.

27. Vanier MC, Bower JM. A comparative survey of automated parameter-search methods for compartmental neural models. Journal of Computational Neuroscience. 1999;7:149.

28. Vogelstein JT, Packer AM, Machado TA, Sippy T, Babadi B, Yuste R, et al. Fast nonnegative deconvolution for spike train inference from population calcium imaging. Journal of Neurophysiology. 2010;104:3691-3704.

29. Druckmann S, Berger TK, Hill S, Schürmann F, Markram H, Segev I. Evaluating automated parameter constraining procedures of neuron models by experimental and surrogate data. Biological Cybernetics. 2008;99:371-379. doi:10.1007/s00422-008-0269-2.

30. van Geit W, Schutter ED, Achard P. Automated neuron model optimization techniques: a review. Biological Cybernetics. 2008;99:241-51.

31. Taylor JD, Winnall S, Nogaret A. Estimation of neuron parameters from imperfect observations. PLoS Computational Biology. 2020;16:1-22. doi:10.1371/journal.pcbi.1008053.

32. Ye J, Rey D, Kadakia N, Eldridge M, Morone UI, Rozdeba P, et al. Systematic variational method for statistical nonlinear state and parameter estimation. Physical Review E. 2015;92:052901. doi:10.1103/PhysRevE.92.052901.

33. Ye J, Kadakia N, Rozdeba PJ, Abarbanel HDI, Quinn JC. Improved variational methods in statistical data assimilation. Nonlinear Processes in Geophysics. 2015;22:205-213. doi:10.5194/npg-22-205-2015.

34. Ye J, Kadakia N, Rozdeba PJ, Abarbanel HDI, Quinn JC. Precision variational approximations in statistical data assimilation. Nonlinear Processes in Geophysics Discussions. 2014;1.

35. Schumann-Bischoff J, Parlitz U. State and parameter estimation using unconstrained optimization. Physical Review E. 2011;84:056214. doi:10.1103/PhysRevE.84.056214.

36. Creveling DR, Gill PE, Abarbanel HDI. State and parameter estimation in nonlinear systems as an optimal tracking problem. Physics Letters A. 2008;372(15):2640 - 2644. doi:10.1016/j.physleta.2007.12.051.

37. Voss HU, Timmer J, Kurths J. Nonlinear dynamical system identification from uncertain and indirect measurements. International Journal of Bifurcation and Chaos. 2004;14:1905-1933. doi:10.1142/S0218127404010345.

38. Pecora LM, Carroll TL. Synchronization in chaotic systems. Physics Review Letters. 1990;64:821.

39. Pecora LM, Carroll TL. Synchronization of chaotic systems. Chaos. 2015;25:097611. doi:10.1063/1.4917383.

40. Abarbanel HDI, Creveling DR, Farsian R, Kostuk M. Dynamical state and parameter estimation. SIAM Journal on Applied Dynamical Systems. 2009;8:1341- 1381 . 
41. Li TY. Numerical solution of multivariate polynomial systems by homotopy continuation methods. Acta Numerica. 1997;6:399-436.

42. Vyasarayani CP, Uchida T, Caravalho A, McPhee J. Parameter identification in dynamic systems using the homotopy optimization approach. Multibody System Dynamics. 2011;26:411-426. doi:10.1007/s11044-011-9260-0.

43. Liberzon D. Calculus of Variations and Optimal Control Theory: A Concise Introduction. Princeton University Press; 2012.

44. Lorenz EN. Predictability: A problem partly solved. In: Proceedings of Seminar on Predictability. vol. 1; 1996.

45. Anthes RA. Data assimilation and initialization of hurricane prediction models. Journal of the Atmospheric Sciences. 1974;31:702-719.

46. Yang SC, Baker D, Li H, Cordes K, Huff M, Nagpal G, et al. Data Assimilation as synchronization of truth and model: Experiments with the three-variable Lorenz System. Journal of the Atmospheric Sciences. 2006;63:2340-2354.

47. Parlitz U. Estimating model parameters from time series by autosynchronization. Physical Review Letters. 1996;76:1232-1235.

48. Hartoyo A, Cadusch PJ, Liley DTJ, Hicks DG. Parameter estimation and identifiability in a neural population model for electro-cortical activity. PLOS Computational Biology. 2019;15(5):1-27. doi:10.1371/journal.pcbi.1006694.

49. Kadakia N, Rey D, Ye J, Abarbanel HDI. Symplectic structure of statistical variational data assimilation. Quarterly Journal of the Royal Meteorological Society. 2017;143(703):756-771. doi:doi.org/10.1002/qj.2962.

50. Bulirsch R, Miele A, Stoer J, Well KH. Optimal Control: Calculus of Variations, Optimal Control Theory and Numerical Methods. Birkhauser; 1993.

51. Pires C, Vautard R, Talagrand O. On extending the limits of variational assimilation in nonlinear chaotic systems. Tellus A. 1996;48:96-121. doi:10.3402/tellusa.v48i1.11634.

52. Koyama S, Paninski L. Efficient computation of the maximum a posteriori path and parameter estimation in integrate-and-fire and more general state-space models. Journal Computational Neuroscience. 2010;29:89-105.

53. Abarbanel HDI, Shirman S, Breen D, Kadakia N, Rey D, Armstrong E, et al. A unifying view of synchronization for data assimilation in complex nonlinear networks. Chaos: An Interdisciplinary Journal of Nonlinear Science. 2017;27(12):126802. doi:10.1063/1.5001816. 